\title{
BMJ Open Sleep duration and apolipoprotein B in metabolically healthy and unhealthy overweight/obese phenotypes: a cross- sectional study in Chinese adults
}

\author{
Huihui Ren, Lu Zhang, Zhelong Liu, Xinrong Zhou, Gang Yuan
}

To cite: Ren H, Zhang L, Liu Z, et al. Sleep duration and apolipoprotein B in metabolically healthy and unhealthy overweight/obese phenotypes: a cross-sectional study in Chinese adults. BMJ Open 2019;9:e023817. doi:10.1136/ bmjopen-2018-023817

- Prepublication history and additional material for this paper are available online. To view these files, please visit the journal online (http://dx.doi. org/10.1136/bmjopen-2018023817).

Received 26 April 2018 Revised 5 January 2019 Accepted 8 January 2019
Check for updates

(C) Author(s) (or their employer(s)) 2019. Re-use permitted under CC BY-NC. No commercial re-use. See rights and permissions. Published by BMJ.

Department of Endocrinology and Metabolism, Tongji

Hospital, Tongji Medical College, Huazhong University of Science and Technology, Wuhan, China

Correspondence to

Dr Gang Yuan;

yuangang88@hotmail.com

\section{ABSTRACT}

Objectives Short sleep duration is independently associated with an increased risk of developing cardiovascular disease; however, the association has not yet been examined in obese populations. We assessed the associations between sleep duration, metabolic phenotype and apolipoprotein variables in a nationally representative Chinese population with overweight/obesity.

Study design Cross-sectional study.

Settings The study conducted in nine provinces of China that vary substantially in geography and economic development.

Patients Data were obtained from 4149 adults with overweight/obesity aged 18 to 94 years from the 2009 China Health and Nutrition Survey. Sleep duration was categorised as $\leq 6,7-8$ or $\geq 9$ hour. Phenotypes were determined based on body mass index and metabolic health status and categorised as metabolically healthy overweight/obesity (MHOO) and metabolically unhealthy overweight/obesity (MU00).

Main outcome measure The outcome variables were elevated apolipoproteins.

Results Compared with MHOO phenotype, MUOO phenotypes were more likely to report shorter sleep duration (12.2\%vs9\%). In the MU00 group, the multivariate-adjusted $\mathrm{OR}(95 \% \mathrm{Cl})$ for elevated apolipoprotein $B$ (apoB) was 1.66 (1.23 to 2.23) for those with $\leq 6$ hours of sleep and 1.12 (0.86 to 1.45$)$ for those with $\geq 9$ hours of sleep, using 7-8 hours of sleep as a reference. Similar results were obtained in the subgroup of subjects who were $\geq 45$ or $<45$ years old, but shorter sleep duration was more strongly associated with elevated apoB in those $<45$ years ( $p$ interaction $=0.023$ ). However, no association was observed in the MHOO phenotype. Conclusions The high prevalence of short sleep duration and its strong association with elevated apoB in adults who are metabolically unhealthy overweight/obese suggest an increased risk of cardiovascular disease in this population. The differences in sleep sufficiency among obese phenotypes may account for the disparities in their cardiovascular outcomes.

\section{INTRODUCTION}

Nutrition transitions and economic changes have led to a considerable increase in the prevalence of obesity worldwide, affecting up

\section{Strengths and limitations of this study}

- The sample size was large and the data are from a geographically diverse household-based cohort in which people are observed in their natural environment.

- The strict and standardised protocols of the cohort regarding the collection of data from questionnaires, anthropometric measures and blood samples was used.

- Sensitivity analyses were used to validate the findings.

- The cross-sectional design does not allow for establishing any causal relationships.

- There may be residual confounding effects, such as using self-reported smoking status and alcohol consumption.

to 600 million people in $2016 .{ }^{1}$ Because of the increased risk for cardiometabolic diseases such as hypertension, dyslipidaemia, diabetes and cardiovascular disease (CVD) in obese individuals, it is not surprising that obesity has become a public health concern. ${ }^{23}$ Interestingly, a proportion of obese individuals express a metabolically healthy obese (MHO) phenotype characterised by relatively low visceral fat, smaller adipocyte size and a more favourable inflammatory profile compared with their metabolically abnormal counterparts. ${ }^{4} 5$ Individuals with metabolically abnormal obesity (MAO) seem to be more susceptible to insulin resistance, impaired glucose tolerance, atherogenic lipid profiles and hypertension, which are considered to be associated with an increased risk for CVD among patients with obesity. However, observations of the cardiometabolic outcomes associated with the MHO phenotype are conflicting. Most studies have reported that individuals with $\mathrm{MHO}$ phenotype have a lower risk for developing cardiometabolic abnormalities, ${ }^{6} 7$ while others have observed a comparable risk of CVD and mortality in 
metabolically healthy and unhealthy overweight/obese phenotypes. ${ }^{89}$

The potential difference in implications of different body size phenotypes for cardiometabolic disease risk may be due to lifestyle factors. Sleep deprivation is widely known to be associated with an increased risk for metabolic diseases ${ }^{10}{ }^{11}$ CVD and mortality ${ }^{12}{ }^{13}$; it predisposes individuals to energy metabolism disturbance, inflammation and dysregulation of neuroendocrine functions, which may contribute to the underlying mechanism of the association between obesity and metabolic characteristics. ${ }^{14}$ Since sleep duration is a modifiable behavioural factor, it is important to understand the potential differences in associations between sleep duration and MHO and MAO phenotypes. Previous studies have evaluated whether sleep duration or sleep quality differ according to MHO and MAO phenotypes. ${ }^{15}{ }^{16}$ However, most of the observations were cross-sectional, had conflicting results or lacked consensus on the definitions of short and long sleep durations.

Additionally, it is unclear how sleep duration may differentially influence the cardiometabolic consequences of these phenotypes. CVD has recently become the leading cause of morbidity and mortality in obesity; additionally, deaths attributable to CVD may be partly explained by sleep debt. An association between sleep duration and CVD risk has been reported in the general population ${ }^{17}{ }^{18}$; however, the association in different obese phenotypes is still unknown. Low apolipoprotein (apo)A1 levels and elevated apoB levels and apoB/A1 ratio have been reported to be independently associated with an increased CVD risk. ${ }^{19}$ A large body of prospective studies have provided evidence that these apolipoprotein variables are superior to conventional cardiovascular risk factors in predicting cardiovascular events. ${ }^{20}{ }^{21}$ This study aimed to address the hypotheses that sleep duration has a differential impact on cardiovascular risk in subjects who are metabolically healthy and unhealthy overweight/ obese. Therefore, using data from the China Health and Nutrition Survey (CHNS) 2009, we explored the relationship between sleep duration and apolipoprotein profile according to overweight/obese phenotypes.

\section{MATERIALS AND METHODS \\ The CHNS}

CHNS is an ongoing longitudinal survey to collect health and nutrition data at the individual, household and community levels. Information collected for each family individual included clinical and sociodemographic characteristics, lifestyle factors, anthropometrics, dietary habits and indicators of health status. The study was conducted from 1989 to 2011 with nine rounds of surveys beginning in eight provinces. A ninth province (Heilongjiang) was added in 1997, and three autonomous cities, Beijing, Shanghai and Chongqing, were added in 2011. The survey used a multistage, random cluster progress to draw samples in the provinces. Counties in the nine provinces were stratified based on income levels (low, middle and high) and a weighted sampling scheme was used to randomly select four counties in each province. Additionally, two cities including the provincial capital and a lower income city were selected. By 2011, the survey provinces covered $47 \%$ of the Chinese population (according to the 2010 census). The details of the design and data collection for the CHNS have been described elsewhere. ${ }^{22}$ The survey was approved by the Institutional Review Committees of the University of North Carolina at Chapel Hill and the National Institute of Nutrition and Food Safety, China Centres for Disease Control and Prevention. All participants provided written informed consent.

\section{Study participants}

Only the 2009 CHNS data were analysed for the purpose of this study because the 2009 survey collected fasting blood samples for the first time. The sample size of the study was estimated based on parameters, such as confidence level $(1-\alpha)$, power of test $(\beta)$ and tolerance $(\delta)$. In the study, the confidence level was $0.95(1-\alpha)$, and the tolerance was 0.3 . According to the literature, ${ }^{22}$ the response rate was $90 \%$, and the pass rate of the questionnaire was predicted to be $70 \%$. Participants were eligible for enrolment if they were 18 years old or older, not pregnant and overweight/obese and had available biomarker data $(n=4372)$. Among them, participants were excluded for the following reasons: missing data on sleep duration $(n=47)$ or metabolic components $(n=62)$ and a history of myocardial infarction and stroke $(n=114)$. A total of 4149 participants (1952 men and 2197 women) between the ages of 18 and 94 years were included in the final analysis.

\section{Data collection and clinical assessments}

A self-administered questionnaire was completed for each individual to collect sociodemographic information including age, smoking status, alcohol consumption, medical history of diabetes, hypertension, myocardial infarction or stroke and use of medications for diabetes, hypertension and dyslipidaemia. Smoking status was categorised as never, former or current smoker. Alcohol drinking status in the previous year was evaluated and categorised as either 'ever' or 'never.'

The body weight of the participants was measured to the nearest $0.1 \mathrm{~kg}$, and height was measured to the nearest $0.1 \mathrm{~cm}$ in subjects in light clothing and without shoes. Body mass index (BMI) was calculated as body weight divided by the square of the height $\left(\mathrm{kg} / \mathrm{m}^{2}\right)$. Overweight/ obesity was defined as a $B M I \geq 23 \mathrm{~kg} / \mathrm{m}^{2}$ based on the WHO criteria for Asia. ${ }^{23}$ The waist circumference (WC) of each subject was measured at the midpoint between the lowest rib and the top of the iliac crest. Trained staff obtained blood pressures for each individual after $5 \mathrm{~min}$ of seated rest using a mercury sphygmomanometer. The right arm was selected. Patients were required not to wear tight cloth; avoid the following activities 1 hour before blood pressure: intense sports or exercise, eating, 
drinking (except water), especially drinks with caffeine; expose under very high/verylow temperature; take medicine which may affect blood pressure; etc. Measures were collected in triplicate, and the average of the three readings was used in the analyses.

\section{Sleep duration assessment}

Sleep duration of all adults was assessed from self-reported questionnaires that asked about the number of hours of sleep obtained in a 24-hour period. For sleep assessment, individuals were asked, 'How many hours each day do you usually sleep, including daytime and nighttime?'. Short sleep duration was defined as $\leq 6$ hours/day, optimal sleep as 7 and 8 hours/day, and long sleep as $\geq 9$ hours/day. Self-reported has been shown to correlated highly with objective measures (Pearson's correlation coefficients, range $0.84-0.95){ }^{24}$

\section{Biochemical assessment}

Fasting (8-12hours overnight) blood samples were obtained from each participant and were separated immediately and frozen at $-86^{\circ} \mathrm{C}$ for subsequent laboratory analysis. Serum glucose was measured by the GOD-PAP method using a Hitachi 7600 analyzer (Randox Laboratories, UK). Serum insulin was measured using radioimmunoassay (North Institute of Bio-Tech, China). Insulin resistance was assessed using the homoeostasis model assessment of insulin resistance (HOMA-IR) defined as follows: fasting plasma glucose (FPG, mmol/L) $\times-$ fasting blood insulin $(\mu \mathrm{U} / \mathrm{mL}) / 22.5$. Serum apoA1 and apoB levels were determined by the immunoturbidimetric method (Randox Laboratories, UK). Serum apoA1 and apoB levels were determined by the immunoturbidimetric method (Randox Laboratories, UK). Decreased apoA1 levels were defined as $<15$ th percentile $(0.85 \mathrm{~g} / \mathrm{L})$, elevated apoB were defined as $\geq 85$ th percentile $(1.3 \mathrm{~g} / \mathrm{L})$ and elevated apoB/apoA1 ratio was defined as $\geq 85$ th percentile. ${ }^{25}$ Low-density and high-density lipoprotein cholesterol (LDL-C and HDL-C, respectively) were measured by the enzymatic method (Kyowa, Japan). Serum triglyceride (TG) concentration and total cholesterol (TC) were measured by the GPO-PAP method and CHOD-PAP method (Kyowa, Japan), respectively. All lipid measurements were performed on the Hitachi 7600 automated analyzer (Hitachi, Tokyo, Japan). Serum high-sensitivity C-reactive protein (hs-CRP) concentrations were measured using the immunoturbidimetric assay (Denka Seiken, Japan).

\section{Definitions of body size phenotypes}

Individuals were classified as metabolically at-risk if they met two or more metabolic abnormalities according to the criteria for the diagnosis of metabolic syndrome recommended by the Adult Treatment Panel (ATP) III of the National Cholesterol Education Programme. ${ }^{26}$ These metabolic components were defined as follows: (1) TG concentration $\geq 150 \mathrm{mg} / \mathrm{dL}$; (2) HDL-C levels $<40 \mathrm{mg} / \mathrm{dL}$ for men and $<50 \mathrm{mg} / \mathrm{dL}$ for women; (3) systolic/diastolic blood pressure $(\mathrm{SBP} / \mathrm{DBP}) \geq 130 / 85 \mathrm{~mm} \mathrm{Hg}$ or antihypertensive medication use; and (4) FPG $\geq 100 \mathrm{mg} / \mathrm{dL}$ or antidiabetes medication use. Individuals with one or none of the abnormal components were classified as metabolically healthy. The following two body size phenotypes were classified based on consideration of both BMI values and the metabolic status: metabolically healthy overweight/obesity (MHOO) (BMI $\geq 23 \mathrm{~kg} / \mathrm{m}^{2}$ and $<2$ metabolic syndrome components) and metabolically unhealthy overweight/obesity (MUOO) (BMI $\geq 23 \mathrm{~kg} / \mathrm{m}^{2}$ and $\geq 2$ metabolic syndrome components).

\section{Patients involvement}

No patients were involved in the development of research question, the outcome measures, the design or implementation of the study. There are no plans about dissemination of the results.

\section{Statistical analysis}

Statistical analyses were performed using SPSS software (V.19; SPSS, Chicago, Illinois, USA). $\mathrm{P}<0.05$ was considered statistically significant. Continuous variables were expressed as the means and SEs, and categorical data were expressed as percentages. Differences in continuous variables between groups were analysed using general linear models, while the differences in categorical variables were analysed using the $\chi^{2}$ test or Fisher's exact test. A Bonferroni post hoc analysis was used for multiple comparisons when necessary. Univariate and multiple logistic regression models were used to determine the independent associations between sleep duration ( $\leq 6$ hours/day, $7-8$ hours/day, $\geq 9$ hours/day) and the variables of apoA1, apoB and the apoB/A1 ratio using the optimal sleep duration of $7-8$ hours/day as the reference category. Multiple logistic regression analysis was performed with low apoA1 levels, high apoB levels and high apoB/apoAl ratios as the dependent variable. The potential confounders entered into the model was based on its significance in univariate analysis $(p<0.15)$ and clinical implication. Models were initially adjusted for age and sex, and were additionally adjusted for potential
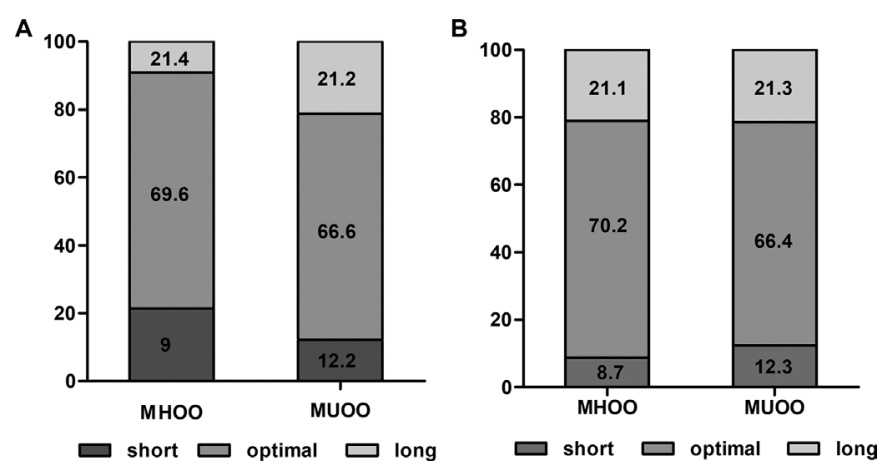

Figure 1 The proportion of sleep duration among the two overweight/obese phenotypes according to the Adult Treatment Panel (ATP) III definition (A) or a previously used definition (B). MHOO, metabolically healthy overweight/ obese; MUOO, metabolically unhealthy overweight/obese. 


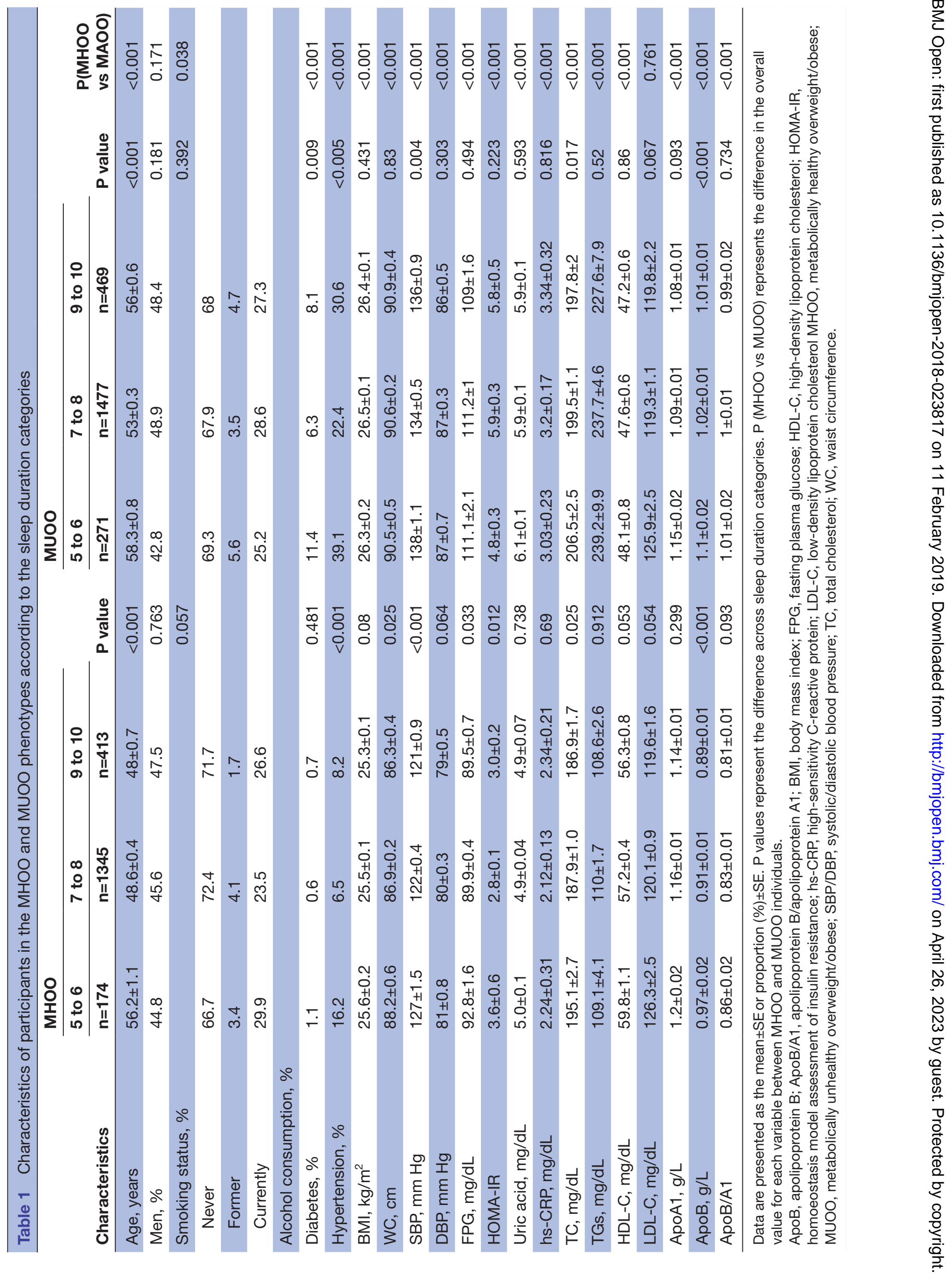


Table 2 ORs and $95 \%$ Cls of decreased apoA1, elevated apoB and elevated apoB/A1 ratio according to the sleep duration categories in the MHOO and MUOO phenotypes*

\begin{tabular}{|c|c|c|c|c|c|c|}
\hline & \multicolumn{4}{|l|}{ MHOO } & \multicolumn{2}{|l|}{ MUOO } \\
\hline & 5 to 6 & 7 to 8 & 9 to 10 & 5 to 6 & 7 to 8 & 9 to 10 \\
\hline \multicolumn{7}{|l|}{ ApoA1 } \\
\hline Model 2 & $1.19(0.67-2.11)$ & - & 0.81 (0.53 to 1.23$)$ & 0.8 (0.55 to 1.15$)$ & - & 1.07 (0.82 to 1.4$)$ \\
\hline Model 3 & $1.22(0.69-2.18)$ & - & 0.81 (0.53 to 1.24$)$ & $0.79(0.54$ to 1.15$)$ & - & $1.04(0.79$ to 1.35$)$ \\
\hline Model 1 & $1.08(0.65-1.8)$ & - & 0.97 (0.67 to 1.4$)$ & 1.85 (1.38 to 2.47$)$ & - & $1.18(0.91$ to 1.53$)$ \\
\hline Model 2 & $0.94(0.56-1.57)$ & - & 0.98 (0.68 to 1.41$)$ & 1.66 (1.23 to 2.23$)$ & - & 1.1 (0.85 to 1.43$)$ \\
\hline Model 3 & $0.91(0.54-1.53)$ & - & $1.02(0.7$ to 1.47$)$ & 1.66 (1.23 to 2.23$)$ & - & $1.12(0.86$ to 1.45$)$ \\
\hline \multicolumn{7}{|l|}{ ApoB/A1 } \\
\hline
\end{tabular}

Data are presented as the OR $(95 \% \mathrm{Cl})$. Model 1 represents a crude model. Model 2 shows the results after adjusting for age and gender. Model 3 further adjusted for smoking habits, alcohol consumption and body mass index.

${ }^{*}$ Compared with the reference category of 7 to 8 hours of sleep. Metabolic health was defined according to the Adult Treatment Panel (ATP) III. ApoA1, apolipoprotein A1; ApoB, apolipoprotein B; MHOO, metabolically healthy overweight/obese; MUOO, metabolically unhealthy overweight/obese.

confounding factors, including smoking habits, alcohol consumption and BMI.

To evaluate potential differential associations of sleep duration by age ( $<45$ vs $\geq 45$ years), sex (male vs female), smoking status (current vs ever/never smoker), alcohol consumption (yes vs no) and medical history of chronic disease (presence vs absence), including diabetes mellitus and hypertension, stratified analyses and interaction tests were performed using logistic regression analysis with elevated apoB as the outcome of interest. ORs with 95\% CIs from logistic regression models were used to assess the associations between sleep duration and apoB level and were shown by forest plots. In sensitivity analyses, we examined the relationship between sleep duration and elevated apoB levels using alternative definitions for metabolic health status used in prior literature. ${ }^{27}$ Metabolically healthy was defined as having one or none of the following metabolic parameters (SBP/ DBP $\geq 130 / 85 \mathrm{~mm} \mathrm{Hg}$ or antihypertensive medication use; TG levels $\geq 150 \mathrm{mg} / \mathrm{L}$; FPG levels $\geq 100 \mathrm{mg} / \mathrm{dL}$ or antidiabetes medication use; HDL-C levels $<40 \mathrm{mg} / \mathrm{dL}$ in men or $<50 \mathrm{mg} / \mathrm{dL}$ in women; HOMA-IR $>90$ th percentile; and hs-CRP $>90$ th percentile).

\section{RESULTS}

MHOO phenotype was observed in $46.6 \%$ ( $\mathrm{n}=1932$ ) of adults who are overweight/obese. The prevalence of short sleep duration was higher $(12.2 \%$ vs $9 \%, \mathrm{p}=0.003)$ and long sleep duration was lower ( $66.6 \%$ vs $69.6 \%, \mathrm{p}=0.003$ ) in the MUOO group compared with the MHOO group (figure 1A).
Both MHOO and MUOO phenotypes with short sleep duration tended to be older, and more likely to have hypertension and diabetes, higher SBP, higher TC and higher apoB (table 1). Additionally, MHOO phenotypes with short sleep duration were more likely to have a higher WC, FPG and HOMA-IR. Compared with MUOO phenotype, MHOO phenotype tended to be younger and non-smokers and to have a more favourable metabolic profile (table 1).

Compared with the prevalence in the MHOO group, a higher prevalence of individuals with decreased apoA1 ( $8.3 \%$ vs $18.9 \%, \mathrm{p}<0.001)$, elevated apoB $(10.2 \%$ vs $20.4 \%$, $\mathrm{p}<0.001)$ and elevated apoB/A1 ratio $(8.9 \%$ vs $22.2 \%$, $\mathrm{p}<0.001)$ was observed in the MUOO group. Of all adults with overweight/obesity, we observed a significant association only between short sleep duration and elevated apoB after adjusting for potential confounders (OR 1.46, 95\% CI 1.14 to 1.88 ) (see online supplementary file 1).

The ORs and 95\% CIs reflecting lower apoA1, higher apoB and higher apoB/apoA1 by sleep duration in MHOO and MUOO phenotypes are shown in table 2. In MUOO phenotype, the ORs for higher apoB levels with short sleep and long sleep compared with optimal sleep were 1.66 (95\% CI 1.23 to 2.23) and 1.12 (95\% CI 0.86 to 1.45 ), respectively ( $\mathrm{p}$ for trend $=0.102$ ), after adjusting for potential confounders. However, no significant associations were observed for sleep duration and apoA1 levels and the apoB:apoA1 ratio in the MUOO phenotype. Additionally, there was no association between sleep duration and apolipoprotein variables in the MHOO phenotype. 
A Characteristic

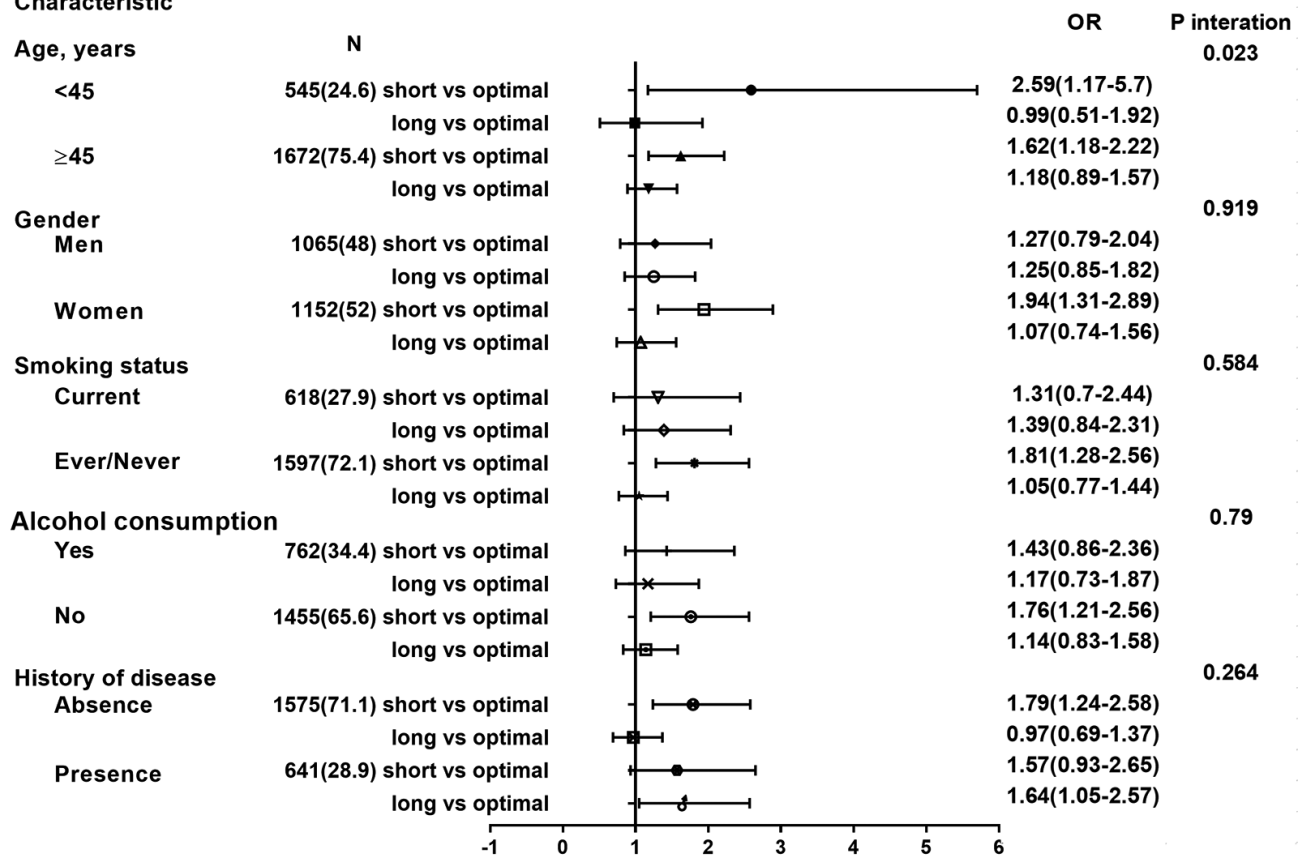

B Characteristic Age, years $<45$

$\geq 45$

Gender

Men

Women

Smoking status Current

Ever/Never

Alcohol consumption Yes

No

History of disease Absence

Presence
N

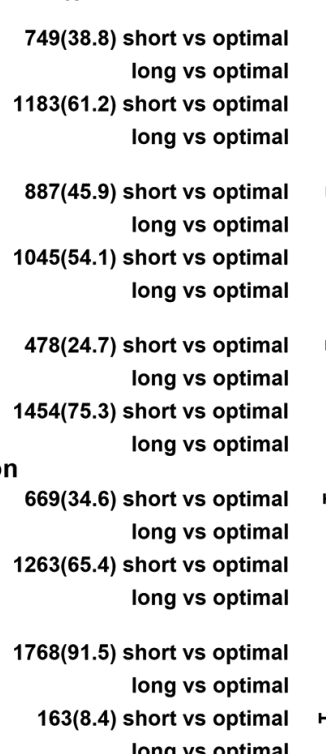

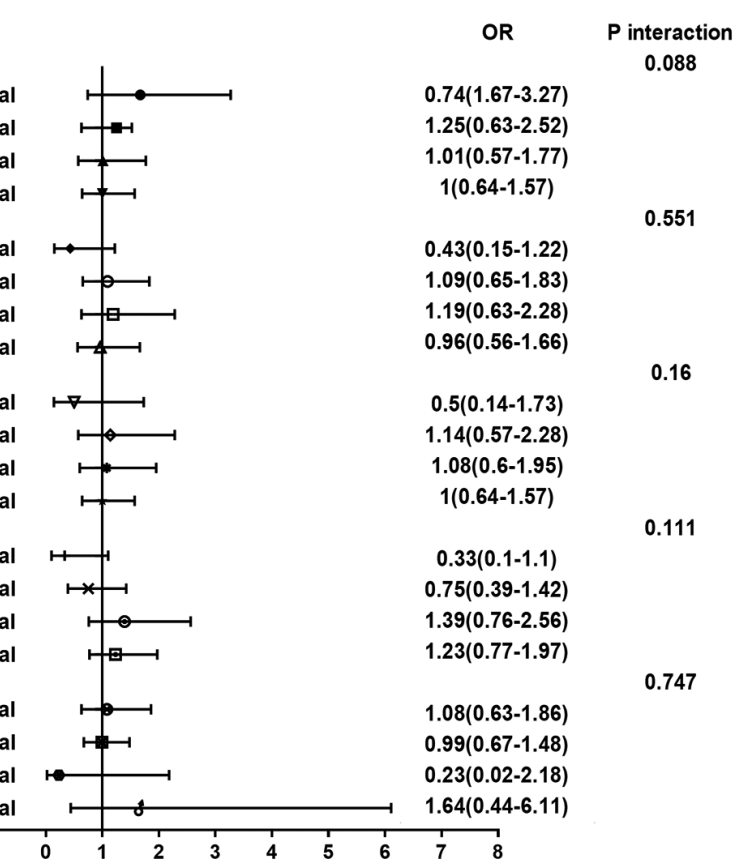

Figure 2 Subgroup analyses of the association between sleep duration and apolipoprotein B in metabolically unhealthy overweight/obese (A) and metabolically healthy overweight/obese (B) phenotypes. Models are adjusted for age, sex and and rural/urban sited, smoking habits, alcohol consumption, educational levels and body mass index, except for the stratifying factor. Presence of disease represents having diabetes mellitus and hypertension.

In stratified analyses for abnormal apoB levels in the MUOO phenotype, the positive association between short sleep and elevated apoB was stronger for individuals younger than 45 years ( $\mathrm{P}$ interaction=0.023) (figure 2a). The association for short sleep duration tended to be stronger among female participants, non-smokers, non-drinkers and those without chronic disease including diabetes mellitus and hypertension; however, these interaction terms were not significant (figure 2A). Similar findings were observed in the MHOO phenotype but did not meet statistical significance (figure 2B).

Additionally, using a different definition of metabolic health, the prevalence of the MHOO phenotype was $43.6 \%(n=1807)$. Similar results were observed in terms of sleep duration (figure 1B) and elevated apoB levels with short sleep duration in MUOO phenotype only (table 3). 
Table 3 ORs and $95 \%$ Cls of decreased apoA1, elevated apoB and elevated apoB/A1 ratio according to the sleep duration categories in the $\mathrm{MHOO}$ and MUOO phenotypes*

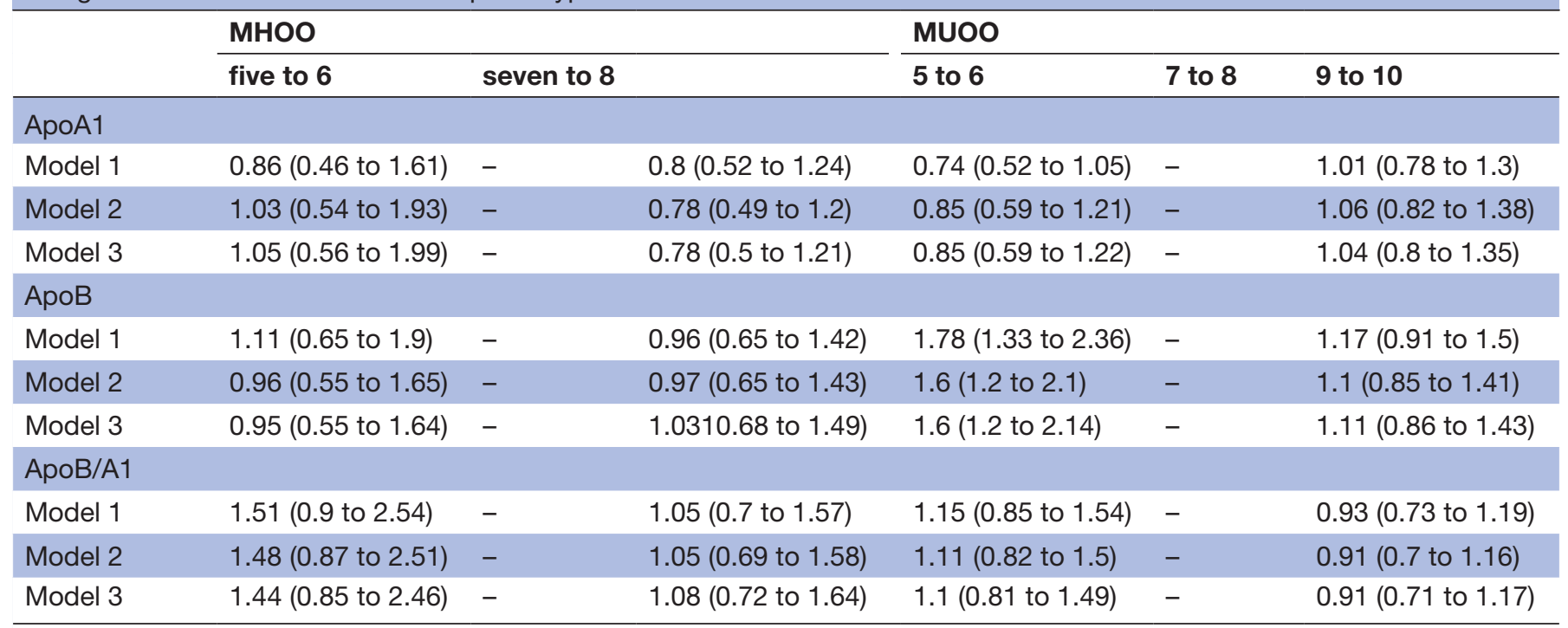

Data are presented as the OR $(95 \% \mathrm{Cl})$. Model 1 represents a crude model. Model 2 shows the results after adjusting for age and gender. Model 3 further adjusted for smoking habits, alcohol consumption and body mass index.

${ }^{*}$ Compared with the reference category of 7 to 8 hours of sleep. Metabolic health was defined according to previous studies. ApoA1, apolipoprotein A1; ApoB, apolipoprotein B; MHOO, metabolically healthy overweight/obese; MUOO, metabolically unhealthy overweight/obese.

\section{DISCUSSION}

In a nationally representative sample of Chinese adults who are overweight/obese, short sleep duration was associated with higher apoB levels in the MUOO phenotype, independent of potential confounders. We observed a $66 \%$ increase in the risk of higher apoB levels in individuals with short sleep duration among MUOO subjects. The association was stronger in individuals who were younger than 45 years old compared with those aged 45 years old or older. However, the adverse association between short sleep duration and elevated apoB was not observed in the MHOO phenotype. To our knowledge, apoB is an independent predictor of CVD. The present study is the first to report a differential association of sleep duration with cardiovascular risk in the MHOO and MUOO phenotypes.

Epidemiological studies have demonstrated an association between decreased sleep duration and an increased prevalence of obesity, ${ }^{28}{ }^{29} \mathrm{CVD}^{30} 31$ and mortality risk. ${ }^{12} \mathrm{~A}$ meta-analysis of 15 prospective studies showed that sleep duration was associated with an increased risk of developing CVD and stroke. ${ }^{17}$ In a prospective study of 10308 participants from the Whitehall II cohort, Tarani $e t a l^{18}$ reported that short sleep duration and sleep disturbance have a combined effect on increasing CVD risk. However, no study has explored the association between sleep duration and cardiovascular risk in the population with overweight/obesity, irrespective of their metabolic health. Our results showed that sleep loss was independently associated with elevated apoB in MUOO phenotype, and this association persisted in our sensitivity analyses. It is well established that apoB is a better predictor of premature coronary artery disease,$^{20}$ fatal myocardial infarction ${ }^{21}$ and coronary artery calcification in midlife ${ }^{32}$ than traditional factors, including TC, TG and LDL-C. Our data appear to confirm the previous findings that short sleep duration increases the risk of incident CVD.

The underlying mechanisms of the adverse effect of short sleep duration on the abnormal functions in MUOO phenotype are largely unclarified. It is known that MUOO phenotype is at high risk of an adverse inflammatory profile, hormonal/metabolic disturbances and excess visceral fat. ${ }^{33}{ }^{34}$ Insufficient sleep-associated metabolic hormones alteration and chronic inflammation may be implicated in the underlying mechanism. Sleep deprivation can significantly affect energy expenditure, weight regulation and inflammatory cytokine levels, ${ }^{35} 36$ which may contribute to the pathogenesis of abnormal apo levels. Sleep loss is also associated with metabolic hormonal alterations, including reduced leptin concentration levels and increased serum ghrelin levels, which can considerably influence lipid metabolism. ${ }^{35}$ Additionally, sleep restriction increases the expression of genes related to immune function, including those for interleukin-8 production and nuclear factor kappa-light-chainenhancer of activated B cells signalling, which could result in chronic, low-level inflammation. ${ }^{37}$ Furthermore, sleep loss was significantly associated with elevated CRP, ${ }^{38}$ an atherogenic dyslipidaemia profile, ${ }^{39} 40$ and insulin resistance, ${ }^{41}$ which are also involved in the pathogenesis of CVD. These observations suggest that sleep deprivation may increase the risk of abnormal apo variables, thereby increasing the risk of abnormal metabolic indices and CVD, or that sleep loss mediates the effect on excess 
CVD risk through abnormal apo variables and metabolic indices in the MUOO phenotype.

Interestingly, the deleterious effect of short sleep duration on cardiovascular risk was more pronounced in those aged 18-44 years in MUOO phenotype. Since adults aged 18-44 years are less likely to report a prevalence of cardiometabolic risk factors, ${ }^{42}$ the increased CVD risk can be mainly attributed to short sleep duration. In addition, those individuals were more likely to work long hours, work in shifts and use electronics, such as cell phones, televisions or computers, all of which can lead to decreased sleep duration. ${ }^{43}$ Among those aged $45 \mathrm{y}$ or older, short sleep duration accounted for only $62 \%$ of the increase in CVD risk, suggesting the synergistic effects of an unfavourable cardiometabolic profile and potentially unhealthy lifestyles. Therefore, more evidence is necessary from prospective studies assessing the effects of short sleep duration on CVD risk in MUOO phenotype while considering disparities by age.

We found no association between short sleep duration and elevated apoB in MHOO phenotype. This finding may suggest that sleep loss alone may not be adequate to increase the cardiovascular risk of those already at lower risk for CVD. It is important to consider that these individuals with MHOO phenotype have favourable metabolic profiles and are, on average, younger than those with MUOO phenotype, less likely to report current smoking and less likely to have hypertension and diabetes. Moreover, the relatively low prevalence of abnormal apoB levels in adults with MHOO phenotype may have limited the investigation of the risk of CVD. In fact, in the univariate analyses, short sleepers had higher FBS, higher insulin resistance, higher SBP and higher apoB levels, though these associations were no longer statistically significant after adjusting for multiple confounders. Previous studies have demonstrated that subjects with MHOO phenotype are at increased risk of adverse long-term outcomes. The prospective cohort North West Adelaide Health Study reported that $\mathrm{MHO}$ was an unstable state and that individuals with MHO had an increased risk of developing diabetes during the 5-10-year follow-up period. ${ }^{43}$ Another prospective population-based study of 3052 Spanish individuals demonstrated that a subset of individuals with MHO progressed from MHOO to MUOO over time. ${ }^{44}$ These data suggested that management of metabolic risk factors should also be recommended to MHO. The longitudinal effect of short sleep on CVD risk in the MHOO phenotype is not well described or understood and requires further investigation.

The strengths of our study include the large sample size and that the data are from a geographically diverse household-based cohort in which people are observed in their natural environment. The strict and standardised protocols of the cohort regarding the collection of data from questionnaires, anthropometric measures and blood samples should also be acknowledged. Moreover, we used sensitivity analyses to validate the findings. Nevertheless, there are several limitations to our study that should be considered. First, the cross-sectional design does not allow for establishing any causal relationships. Longterm prospective cohort studies are needed. Second, our findings are based on self-reported sleep duration. However, self-reported sleep duration has been validated by polysomnography or actigraphy in previous studies and has been shown to be as accurate as these objective measures. ${ }^{24}$ Additionally, the questionnaires in the present study were not validated, and some variables were self-reported which could cause systematic bias. Thus, in every round, the project teams supervised and conducted quality inspections to make appropriate adjustments to some questions. Finally, although we adjusted for multiple potential confounders, there may be residual confounding effects, such as using self-reported smoking status, alcohol consumption and sleep apnoea. Note that sleep apnoea was reported to be associated with multiple cardiometabolic diseases, which may mediate the association of sleep duration and apolipoprotein variables.

In conclusion, based on a nationally representative Chinese adult population, our results suggest that short sleep duration is associated with a higher risk of CVD exclusively in the MUOO phenotype. Sleep improvement may be particularly beneficial both for overweight and obesity with metabolic abnormalities. The lack of an adverse association between sleep duration and CVD risk in MHOO phenotype may suggest that future prospective cohort studies are needed.

Acknowledgements We would like to acknowledge the China Health and Nutrition Survey, supported by the NIH (R01-HD30880, DK056350 and R01HD38700) and the National Institute of Nutrition and Food Safety, China Centers for Disease Control and Prevention, Carolina Population Center, the University of North Carolina at Chapel Hill and the Fogarty International Center for providing the data used in this study. We also thank the China-Japan Friendship Hospital and the Ministry of Health for supporting the CHNS 2009 surveys.

Contributors GY designed the research study. HR, LZ, ZL, and XZ conducted the study. HR, LZ, ZL, and XZ analysed the data. HR wrote the first draft. All authors read and approved the final manuscript.

Funding This work was supported by a grant from the National Natural Science Foundation of China (№. 81770817, GY).

Competing interests None declared.

Patient consent Obtained.

Ethics approval The survey was approved by the Institutional Review Committees of the University of North Carolina at Chapel Hill and the National Institute of Nutrition and Food Safety, China Centers for Disease Control and Prevention.

Provenance and peer review Not commissioned; externally peer reviewed.

Data sharing statement The data sets used for this study are available on request from the China Health and Nutrition Survey database (www.cpc.unc.edu/projects/ china/).

Open access This is an open access article distributed in accordance with the Creative Commons Attribution Non Commercial (CC BY-NC 4.0) license, which permits others to distribute, remix, adapt, build upon this work non-commercially, and license their derivative works on different terms, provided the original work is properly cited, appropriate credit is given, any changes made indicated, and the use is non-commercial. See: http://creativecommons.org/licenses/by-nc/4.0/.

\section{REFERENCES}

1. WHO. Obesity and overweight. http://www.who.int/mediacentre/ factsheets/fs311/en/ (Accessed 6 Dec 2017). 
2. Eckel RH, Kahn R, Robertson RM, et al. Preventing cardiovascular disease and diabetes: a call to action from the American diabetes association and the american heart association. Diabetes Care 2006;29:1697-9.

3. Hossain P, Kawar B, El Nahas M. Obesity and diabetes in the developing world-a growing challenge. $N$ Engl J Med 2007;356:213-5.

4. Karelis AD, Faraj M, Bastard JP, et al. The metabolically healthy but obese individual presents a favorable inflammation profile. J Clin Endocrinol Metab 2005;90:4145-50.

5. Primeau V, Coderre L, Karelis AD, et al. Characterizing the profile of obese patients who are metabolically healthy. Int $J$ Obes 2011;35:971-81.

6. Dobson R, Burgess MI, Sprung VS, et al. Metabolically healthy and unhealthy obesity: differential effects on myocardial function according to metabolic syndrome, rather than obesity. Int $J$ Obes 2016;40:153-61.

7. Ogorodnikova AD, Kim M, McGinn AP, et al. Incident cardiovascular disease events in metabolically benign obese individuals. Obesity 2012;20:651-9.

8. Kramer CK, Zinman B, Retnakaran R. Are metabolically healthy overweight and obesity benign conditions?: a systematic review and meta-analysis. Ann Intern Med 2013;159:758-69.

9. Fan J, Song Y, Chen Y, et al. Combined effect of obesity and cardio-metabolic abnormality on the risk of cardiovascular disease: a meta-analysis of prospective cohort studies. Int J Cardiol 2013;168:4761-8.

10. Schmid SM, Hallschmid M, Schultes B. The metabolic burden of sleep loss. Lancet Diabetes Endocrinol 2015;3:52-62.

11. Chaput JP, McNeil J, Després JP, et al. Short sleep duration as a risk factor for the development of the metabolic syndrome in adults. Prev Med 2013;57:872-7.

12. Xiao Q, Keadle SK, Hollenbeck AR, et al. Sleep duration and total and cause-specific mortality in a large US cohort: interrelationships with physical activity, sedentary behavior, and body mass index. Am $J$ Epidemiol 2014;180:997-1006.

13. Kronholm E, Laatikainen T, Peltonen M, et al. Self-reported sleep duration, all-cause mortality, cardiovascular mortality and morbidity in Finland. Sleep Med 2011;12:215-21.

14. Porkka-Heiskanen T, Zitting KM, Wigren HK. Sleep, its regulation and possible mechanisms of sleep disturbances. Acta Physiol 2013;208:311-28.

15. Ryu JY, Lee JS, Hong HC, et al. Association between body size phenotype and sleep duration: Korean National Health and Nutrition Examination Survey V (KNHANES V). Metabolism 2015;64:460-6.

16. Kanagasabai T, Dhanoa R, Kuk JL, et al. Association between sleep habits and metabolically healthy obesity in adults: a cross-sectional study. J Obes 2017;2017:1-7.

17. Cappuccio FP, Cooper D, D'Elia L, et al. Sleep duration predicts cardiovascular outcomes: a systematic review and meta-analysis of prospective studies. Eur Heart J 2011;32:1484-92.

18. Chandola T, Ferrie JE, Perski A, et al. The effect of short sleep duration on coronary heart disease risk is greatest among those with sleep disturbance: a prospective study from the Whitehall II cohort. Sleep 2010;33:739-44.

19. Thompson A, Danesh J. Associations between apolipoprotein B, apolipoprotein $\mathrm{Al}$, the apolipoprotein $\mathrm{B} / \mathrm{Al}$ ratio and coronary heart disease: a literature-based meta-analysis of prospective studies. $J$ Intern Med 2006;259:481-92.

20. Kwiterovich $\mathrm{PO}$, Coresh J, Smith $\mathrm{HH}$, et al. Comparison of the plasma levels of apolipoproteins $B$ and $A-1$, and other risk factors in men and women with premature coronary artery disease. $A m ~ J$ Cardiol 1992;69:1015-21.

21. Walldius G, Jungner I, Holme I, et al. High apolipoprotein B, low apolipoprotein $A-I$, and improvement in the prediction of fatal myocardial infarction (AMORIS study): a prospective study. Lancet 2001;358:2026-33

22. Popkin BM, Du S, Zhai F, et al. Cohort profile: the China health and nutrition survey-monitoring and understanding socio- economic and health change in China, 1989-2011. Int J Epidemiol 2010;39:1435-40.

23. WHO Expert Consultation. Appropriate body-mass index for Asian populations and its implications for policy and intervention strategies. Lancet 2004;363:157-63.

24. Signal TL, Gale J, Gander PH. Sleep measurement in flight crew: comparing actigraphic and subjective estimates to polysomnography. Aviat Space Environ Med 2005;76:1058-63.

25. Muntner P, Hamm LL, Kusek JW, et al. The prevalence of nontraditional risk factors for coronary heart disease in patients with chronic kidney disease. Ann Intern Med 2004;140:9-17.

26. Jama285. Executive summary of the third report of The National Cholesterol Education Program (NCEP) Expert panel on detection, evaluation, and treatment of high blood cholesterol (adult treatment panel III), 2001:2486-97.

27. Park YM, Steck SE, Fung TT, et al. Mediterranean diet and mortality risk in metabolically healthy obese and metabolically unhealthy obese phenotypes. Int J Obes 2016;40:1541-9.

28. Jean-Louis G, Youngstedt S, Grandner M, et al. Unequal burden of sleep-related obesity among black and white Americans. Sleep Health 2015;1:169-76.

29. Wu Y, Zhai L, Zhang D. Sleep duration and obesity among adults: a meta-analysis of prospective studies. Sleep Med 2014;15:1456-62.

30. Wolff B, Völzke H, Schwahn C, et al. Relation of self-reported sleep duration with carotid intima-media thickness in a general population sample. Atherosclerosis 2008;196:727-32.

31. Jackson CL, Redline S, Emmons KM. Sleep as a potential fundamental contributor to disparities in cardiovascular health. Annu Rev Public Health 2015;36:417-40.

32. Wilkins JT, Li RC, Sniderman A, et al. Discordance between apolipoprotein $B$ and LDL-Cholesterol in young adults predicts coronary artery calcification: the CARDIA study. J Am Coll Cardiol 2016;67:193-201.

33. Hwang Y-C, Hayashi T, Fujimoto WY, et al. Visceral abdominal fat accumulation predicts the conversion of metabolically healthy obese subjects to an unhealthy phenotype. Int J Obes 2015;39:1365-70.

34. Jung $\mathrm{CH}$, Lee WJ, Song $\mathrm{KH}$. Metabolically healthy obesity: a friend or foe? Korean J Intern Med 2017;32:611-21.

35. Taheri S, Lin L, Austin D, et al. Short sleep duration is associated with reduced leptin, elevated ghrelin, and increased body mass index. PLoS Med 2004;1:e62.

36. Patel SR, Malhotra A, White DP, et al. Association between reduced sleep and weight gain in women. Am J Epidemiol 2006;164:947-54.

37. Aho V, Ollila HM, Rantanen V, et al. Partial sleep restriction activates immune response-related gene expression pathways: experimental and epidemiological studies in humans. PLoS One 2013;8:e77184.

38. Chiang JK. Short duration of sleep is associated with elevated high-sensitivity C-reactive protein level in Taiwanese adults: a crosssectional study. J Clin Sleep Med 2014;10:743-9.

39. Gangwisch JE, Malaspina D, Babiss LA, et al. Short sleep duration as a risk factor for hypercholesterolemia: analyses of the Nationa longitudinal study of adolescent health. Sleep 2010;33:956-61.

40. Kaneita Y, Uchiyama M, Yoshiike N, et al. Associations of usual sleep duration with serum lipid and lipoprotein levels. Sleep 2008;31:645-52.

41. Liu A, Kushida CA, Reaven GM. Habitual shortened sleep and insulin resistance: an independent relationship in obese individuals. Metabolism 2013;62:1553-6.

42. Liu Y, Wheaton AG, Chapman DP, et al. Sleep duration and chronic diseases among U.S. adults age 45 years and older: evidence from the 2010 Behavioral Risk Factor Surveillance System. Sleep 2013;36:1421-7

43. Luckhaupt SE, Tak S, Calvert GM. The prevalence of short sleep duration by industry and occupation in the National Health Interview Survey. Sleep 2010;33:149-59.

44. Schröder H, Ramos R, Baena-Díez JM, et al. Determinants of the transition from a cardiometabolic normal to abnormal overweight/obese phenotype in a Spanish population. Eur J Nutr 2014;53:1345-53. 